See discussions, stats, and author profiles for this publication at: https://www.researchgate.net/publication/330189656

Measuring radical diffusion in viscous liquids by electron paramagnetic resonance

Article in Journal of Molecular Liquids · March 2019

DOI: 10.1016/j.molliq.2019.01.006

CITATION

1

2 authors, including:

Dalibor Merunka

Ruđer Bošković Institute

29 PUBLICATIONS 146 CITATIONS

SEE PROFILE
READS

66 


\section{Measuring Radical Diffusion in Viscous Liquids by Electron Paramagnetic Resonance}

$$
\text { Dalibor Merunka }{ }^{\dagger} * * \text { and Miroslav Peric }{ }^{\ddagger}
$$

${ }^{\dagger}$ Division of Physical Chemistry, Ruđer Bošković Institute, Bijenička cesta 54, HR-10000 Zagreb, Croatia, E-mail: merunka@irb.hr

${ }^{\ddagger}$ Department of Physics and Astronomy and The Center for Biological Physics, California State University at Northridge, Northridge, California 91330, United States,

E-mail: miroslav.peric@ @ csun.edu

October 23, 2018

*Corresponding author. E-mail: merunka@irb.hr, Tel: +3851-4561-136 


\begin{abstract}
Relative diffusion of free radicals in solution modulates the Heisenberg spin exchange and dipoledipole interactions among them, which affects their electron paramagnetic resonance (EPR) spectra. The radical concentration dependence of EPR parameters can, in turn, give information about radical diffusivity in a liquid. We studied the diffusivities of the ${ }^{14} \mathrm{~N}$ - and ${ }^{15} \mathrm{~N}$-labeled perdeuterated TEMPONE radicals at various temperatures in three viscous liquids: 1-ethyl-3-methylimidazolium bis(trifluoromethylsulfonyl)imide ionic liquid, propylene carbonate, and ethylene glycol. By fitting EPR spectra at various radical concentrations, we obtained the concentration coefficients of EPR parameters. The concentration coefficients were related to the radical diffusivity by solving the kinetic equations for the spin evolution of a radical pair, considering the radicals as continuously diffusing spherical objects in the hard-core pair potential. We tested the method by comparing the calculated radical diffusivities of isotopically substituted TEMPONE radicals. Temperature dependences of radical diffusivities were discussed in terms of the Stokes-Einstein relation. Additionally, the radical diffusivities were compared to the self-diffusivities of the studied liquids. At lower temperatures, the radical diffusivities follow the self-diffusivities, while at the higher temperatures, the radical diffusivities start deviating from the self-diffusivities.
\end{abstract}

Keywords: molecular liquids; ionic liquids; electron paramagnetic resonance; nitroxide radicals; spin interactions; tracer diffusivity; 


\section{Introduction}

Diffusion of host molecules (self-diffusion) and diluted guest molecules (tracer diffusion) in neutral molecular liquids and ionic liquids have attracted great attention due to theoretical and practical interests [1-5]. By using electron paramagnetic resonance (EPR) spectroscopy, one can detect free radicals in a liquid solution, as well as obtain information on their translational motion. This is possible because the relative motion of radicals modulates the Heisenberg spin exchange (HSE) and dipole-dipole (DD) spin interactions between them, which in turn changes the shape of the EPR spectrum. The change of the shape of the EPR spectrum depends on the radical concentration $C$ and the diffusion coefficient (diffusivity) of radical $D[6,7]$. Since this modulation induces an additional dephasing of the radical's magnetization and broadens the EPR lines, the effect of spin interactions has traditionally been studied by measuring the concentration-induced broadening of the EPR lines [6,8-13]. Although the line-broadening method has been applied to study radical diffusion in liquids, liquid crystals, biological systems, porous hosts, and other systems $[6,8-13]$, the method is most effective in non-viscous systems, where the diffusion is fast enough that the line broadening due to the HSE interaction dominates over that due to the DD interaction [8]. While the HSE broadening is proportional to the spin exchange frequency, which linearly increases with both $C$ and $D$, the DD broadening increases with decreasing diffusivity, which makes the linebroadening method insensitive to the changes in diffusivity in viscous systems.

In a more advanced study of the effects of the spin interactions on EPR parameters [14], the spin exchange broadened EPR spectra of a nitroxide radical were successfully fitted to the sum of Lorentzian absorption and dispersion lines (absorption-dispersion function) by using a nonlinear least-squares fitting method. The fitting method provided two additional EPR parameters that depend on the spin exchange frequency: (i) the ratio between the amplitudes of dispersion and absorption components, and (ii) the absorption nitrogen hyperfine splitting $A_{a b s}$ calculated from the positions of 
the outer absorption lines $[14,15]$. The spin coherence transfer between hyperfine lines, which is induced by HSE and DD interactions, was predicted to cause the appearance of dispersion components in the EPR spectra [6,7]. The spin coherence transfer together with the mechanism that changes the nitrogen hyperfine splitting $A$ due to the HSE interaction and a finite encounter time of colliding radicals was also predicted to affect $A_{a b s}[7,16]$. In the EPR studies of the stable nitroxide radical, perdeuterated 2,2,6,6-tetramethyl-4-oxopiperidine-1-oxyl $\left({ }^{14} \mathrm{~N}\right.$-pDTEMPONE) in squalane and water, a method to separate the effects of HSE and DD interactions on EPR parameters was proposed [17,18]. The HSE-DD separation method applies approximate relations for the spin coherence transfer and spin dephasing due to the DD interaction using the theoretical analysis derived in Ref. [7]. The absorption-dispersion function fitting and HSE-DD separation method were improved in a follow-up EPR study of ${ }^{14} \mathrm{~N}$-pDTEMPONE and ${ }^{15} \mathrm{~N}$-labeled pDTEMPONE $\left({ }^{15} \mathrm{~N}\right.$ pDTEMPONE) diffusion in water [19]. The EPR spectra of both radicals were fitted to the original spectral function derived from the modified Bloch equations in the presence of HSE and DD interactions. The original function fitting method provides the original EPR parameters directly from the Bloch equations, unlike the absorption-dispersion function fitting method $[14,15]$. However, both fitting methods give the same results after suitable transformations of the EPR parameters obtained by the latter method [19]. The HSE-DD separation method is further improved by iterative solving of the kinetic equations for the spin evolution of two radicals moving according to the continuous diffusion model and interacting by HSE and DD interactions [19]. The obtained relations for the concentration coefficients of spin dephasing, spin coherence transfer, and hyperfine splitting were then used to calculate the diffusivities of radicals.

Here, we apply these experimental and theoretical methods to study the diffusivities of the

${ }^{14} \mathrm{~N}$ - and ${ }^{15} \mathrm{~N}$-pDTEMPONE radicals at various temperatures in three different viscous liquids: 1 ethyl-3-methylimidazolium bis(trifluoromethylsulfonyl)imide ionic liquid, glass forming liquid propylene carbonate, and hydrogen-bonding liquid ethylene glycol. The choice of these two radicals 
is useful for testing the HSE-DD separation methods since these radicals' EPR spectra are very different while their size and diffusivity are the same.

\section{Materials and Methods}

\subsection{Materials and EPR spectroscopy}

The spin probes ${ }^{15} \mathrm{~N}$-pDTEMPONE (98 atom \% D, 99 atom $\%{ }^{15} \mathrm{~N}$ ) and ${ }^{14} \mathrm{~N}-\mathrm{pDTEMPONE}$ (99 atom \% D) were purchased from CDN Isotopes and used as received. The ionic liquid 1-ethyl-3methylimidazolium bis(trifluoromethylsulfonyl)imide $\left(\mathrm{C}_{2} \mathrm{C}_{1} \mathrm{ImTFSI}\right)$ of $99 \%$ purity was purchased from IOLITEC. It was dried under vacuum for three days before use. Propylene carbonate (PC) and ethylene glycol (EG), both of 99\% purity, were purchased from Alfa Aesar and used as received. The structures of the spin probe and the solvents are shown in Figure 1.

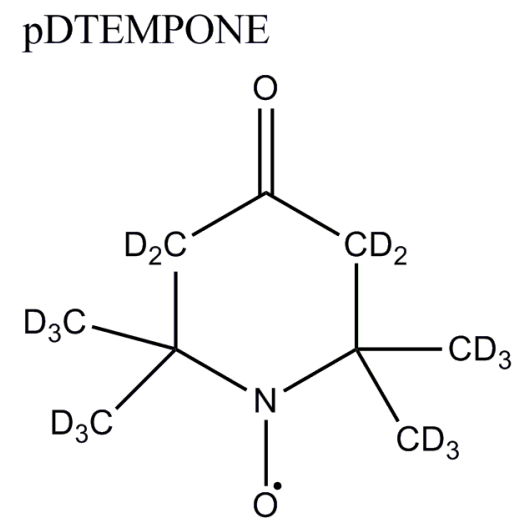

$\mathrm{PC}$

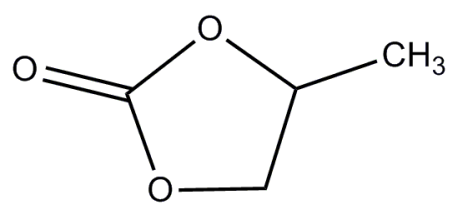

$$
\mathrm{C}_{2} \mathrm{C}_{1} \operatorname{ImTFSI}
$$<smiles>CC[n+]1ccn(C)c1</smiles><smiles>O=S(=O)([O-])NS(=O)(=O)C(F)(F)F</smiles>

EG

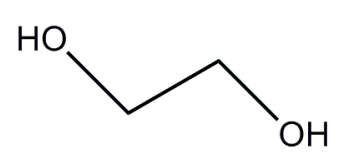

Figure 1. Chemical structures of pDTEMPONE, $\mathrm{C}_{2} \mathrm{C}_{1}$ ImTFSI, PC, and EG.

Stock solutions of $36 \mathrm{mM}$ of ${ }^{15} \mathrm{~N}$ - and ${ }^{14} \mathrm{~N}$-pDTEMPONE were prepared by weight in all solvents. The stock solutions were then diluted to 12 concentrations of pDTEMPONE (from $3 \mathrm{mM}$ to 
$36 \mathrm{mM}$ in steps of $3 \mathrm{mM}$ ), and the exact concentrations were determined by weighing. Just prior to EPR measurements, the samples were drawn into 5 - $\mu \mathrm{L}$ capillaries (radius $\approx 150 \mu \mathrm{m}$ ) and sealed at the lower end by Haematocrit sealing compound, while the upper end was left open. EPR spectra were recorded with a Varian E-109 X-band spectrometer upgraded with a Bruker microwave bridge and a Bruker high-Q cavity. The sample temperature, controlled by a Bruker variable temperature unit, was held stable within $\pm 0.2 \mathrm{~K}$ and measured with a thermocouple using an Omega temperature indicator. The thermocouple tip was always positioned at the top of the active region of the EPR cavity to avoid reducing the cavity quality factor. All samples were measured in steps of $5 \mathrm{~K}$ in various temperature ranges depending on the solvent. The radical concentrations were corrected for each measured temperature using the temperature dependences of solvents' densities from literature. The EPR spectra were acquired with a sweep time of $20 \mathrm{~s}$, microwave power of $0.5 \mathrm{~mW}$, time constant of $16 \mathrm{~ms}$, modulation amplitude of $0.1 \mathrm{G}$, and sweep width of $50 \mathrm{G}$.

\subsection{Fitting EPR spectra and determining the concentration coefficients}

The experimental EPR spectrum $S(B)=d R(B) / d B$ is the first derivative of the absorption EPR signal $R(B)$ with respect to the applied magnetic field $B$. Since the EPR spectra of nitroxide radicals exhibit $2 I+1$ hyperfine lines, where the spin of the nitrogen nucleus is $I=1 / 2$ for ${ }^{15} \mathrm{~N}$ and $I=1$ for ${ }^{14} \mathrm{~N}$, the ${ }^{15} \mathrm{~N}$ - and ${ }^{14} \mathrm{~N}$-labeled radicals exhibit two and three hyperfine lines, respectively (Fig. 2a-b). The original spectral function for the absorption EPR spectra of ${ }^{15} \mathrm{~N}$ - and ${ }^{14} \mathrm{~N}$-labeled radicals interacting by HSE and DD interactions was obtained from the modified Bloch equations [7,19]. It has the form:

$$
R(B)=J_{0} \operatorname{Re}\left[\frac{G(B)}{1-\Lambda G(B)}\right] ; G(B)=\sum_{k=1}^{2 I+1} \frac{1}{z_{k}+\Lambda+i\left(B-B_{0}\right)},
$$


where $J_{0}$ is a constant, while the other parameters having the magnetic field units are: the coherence transfer rate $\Lambda$, the central field line position of the spectrum $B_{0}$, and the $k$-th hyperfine line parameters $z_{k}$. The line parameters have the forms:

$z_{1}=\Gamma_{1}-i A / 2 ; z_{2}=\Gamma_{2}+i A / 2$

for the ${ }^{15} \mathrm{~N}$-labeled radical and the forms:

$z_{1}=\Gamma_{1}-i(A+S / 3) ; z_{2}=\Gamma_{2}+2 i S / 3 ; z_{3}=\Gamma_{3}+i(A-S / 3)$

for the ${ }^{14} \mathrm{~N}$-labeled radical, where $\Gamma_{k}$ is the spin dephasing rate or the linewidth of $k$-th line, $A$ is the nitrogen hyperfine splitting, and $S$ is a small relative second-order hyperfine shift.

Experimental EPR spectra were transferred to a personal computer and fitted to the first derivative of $R(B)$ defined by Eqs. (1). The fitting procedure was performed using the nonlinear regression command in the program package Mathematica. The experimental spectra of 3 and 36 $\mathrm{mM}$ solutions at $60^{\circ} \mathrm{C}$ are shown in Fig. 2a-b, together with the fitting curves and residuals. The fits are quite good, as can be seen from the small values of the residuals, which are dominated by the weak satellite lines due to ${ }^{13} \mathrm{C}$ nuclei.

The concentration-induced increase of spin dephasing rates and the corresponding broadening of EPR lines can be clearly seen in Fig. 2a-b. We define the average linewidth of hyperfine lines as $\Gamma=\left(\Gamma_{1}+\Gamma_{2}\right) / 2$ for ${ }^{15} \mathrm{~N}$ - and $\Gamma=\left(\Gamma_{1}+\Gamma_{2}+\Gamma_{3}\right) / 3$ for ${ }^{14} \mathrm{~N}$-pDTEMPONE, whose calculated values are pltted in Fig. 2c-d, together with the fitted values of $\Lambda$ and $A$. The appearance of dispersion components in the hyperfine lines due to the spin coherence transfer can be seen in Fig.2a-b. A positive coherence transfer rate lifts up the low field line and pushes down the high field line [14,15], which can be clearly seen for the $C=36 \mathrm{mM}$ sample. The coherence transfer rate between the two transitions (lines) of ${ }^{15} \mathrm{~N}$-pDTEMPONE is a well-defined quantity $\Lambda=\Lambda(a)$ depending on the frequency difference between the lines, which is equal to the hyperfine coupling constant $a=\gamma_{e} A$, 
where $\gamma_{e}=g \mu_{\mathrm{B}} / \hbar$ is the electron gyromagnetic ratio ( $g$ is the radical g-factor and $\mu_{\mathrm{B}}$ is the Bohr magneton). Since the frequency differences between the ${ }^{14} \mathrm{~N}$-pDTEMPONE transitions are given by $a$ and $2 a$, neglecting a small second-order shift, the coherence transfer rate between the neighbor lines $\Lambda(a)$ is generally different from that between the outer lines $\Lambda(2 a)$. The analysis showed that this difference is so small that it cannot be extracted by fitting and that the coherence transfer rate obtained from Eq. (1) corresponds to the average coherence transfer over different lines, i.e. $\Lambda=2 \Lambda(a) / 3+\Lambda(2 a) / 3$.
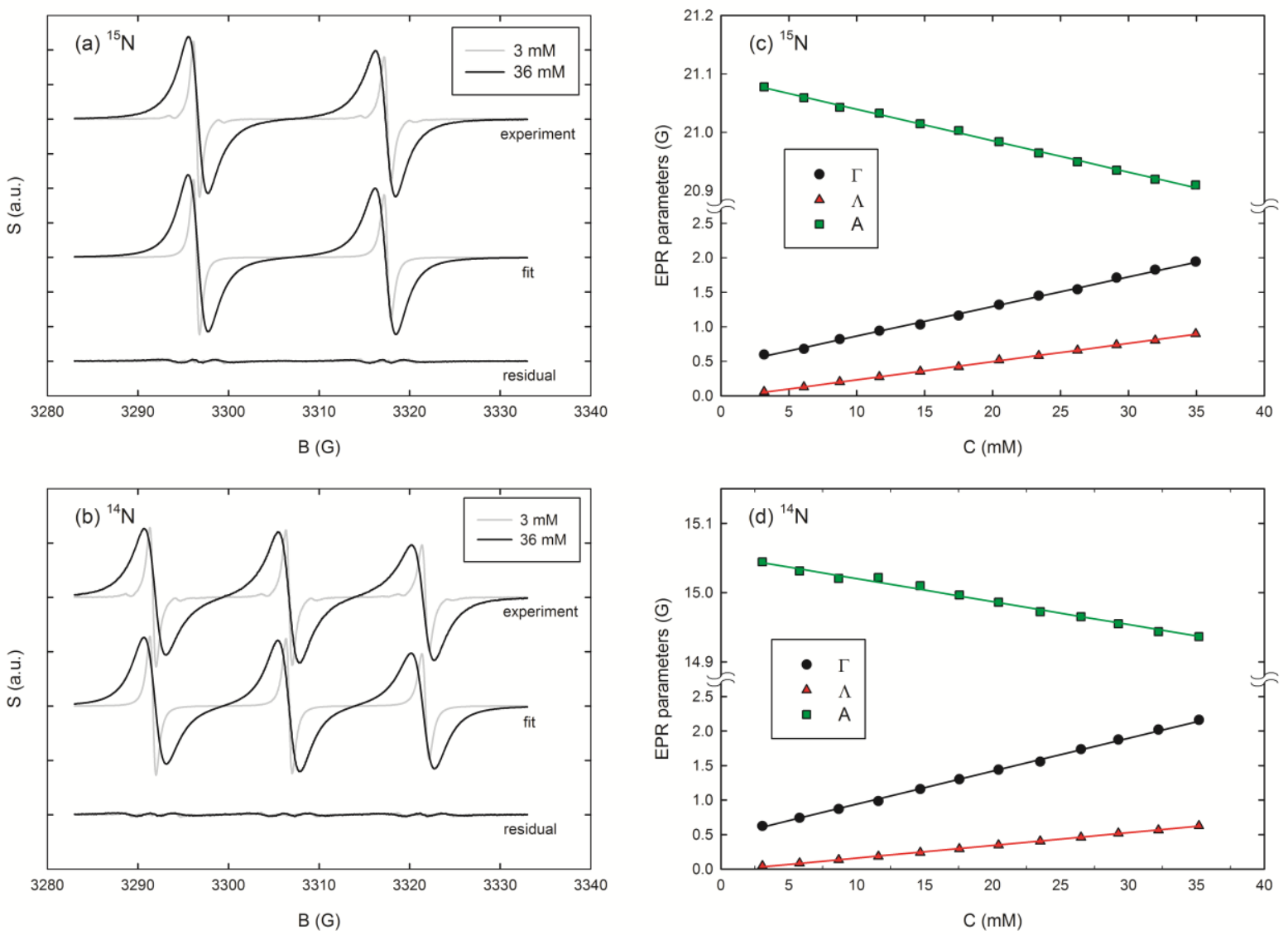

Figure 2. EPR parameters from the fitting procedure. The experimental EPR spectra, fitting curves, and residuals of 3 and $36 \mathrm{mM}$ nitroxide solutions of (a) ${ }^{15} \mathrm{~N}$ - and (b) ${ }^{14} \mathrm{~N}-\mathrm{pDTEMPONE}$ in $\mathrm{C}_{2} \mathrm{C}_{1} \operatorname{ImTFSI}$ at $60^{\circ} \mathrm{C}$. The fitted values of average spin dephasing rate $\Gamma$, coherence transfer rate $\Lambda$, and nitrogen hyperfine splitting $A$ as a function of concentration for (c) ${ }^{15} \mathrm{~N}-$ and (d) ${ }^{14} \mathrm{~N}-$ pDTEMPONE in $\mathrm{C}_{2} \mathrm{C}_{1}$ ImTFSI at $60^{\circ} \mathrm{C}$. The lines are fits to linear concentration dependences. 

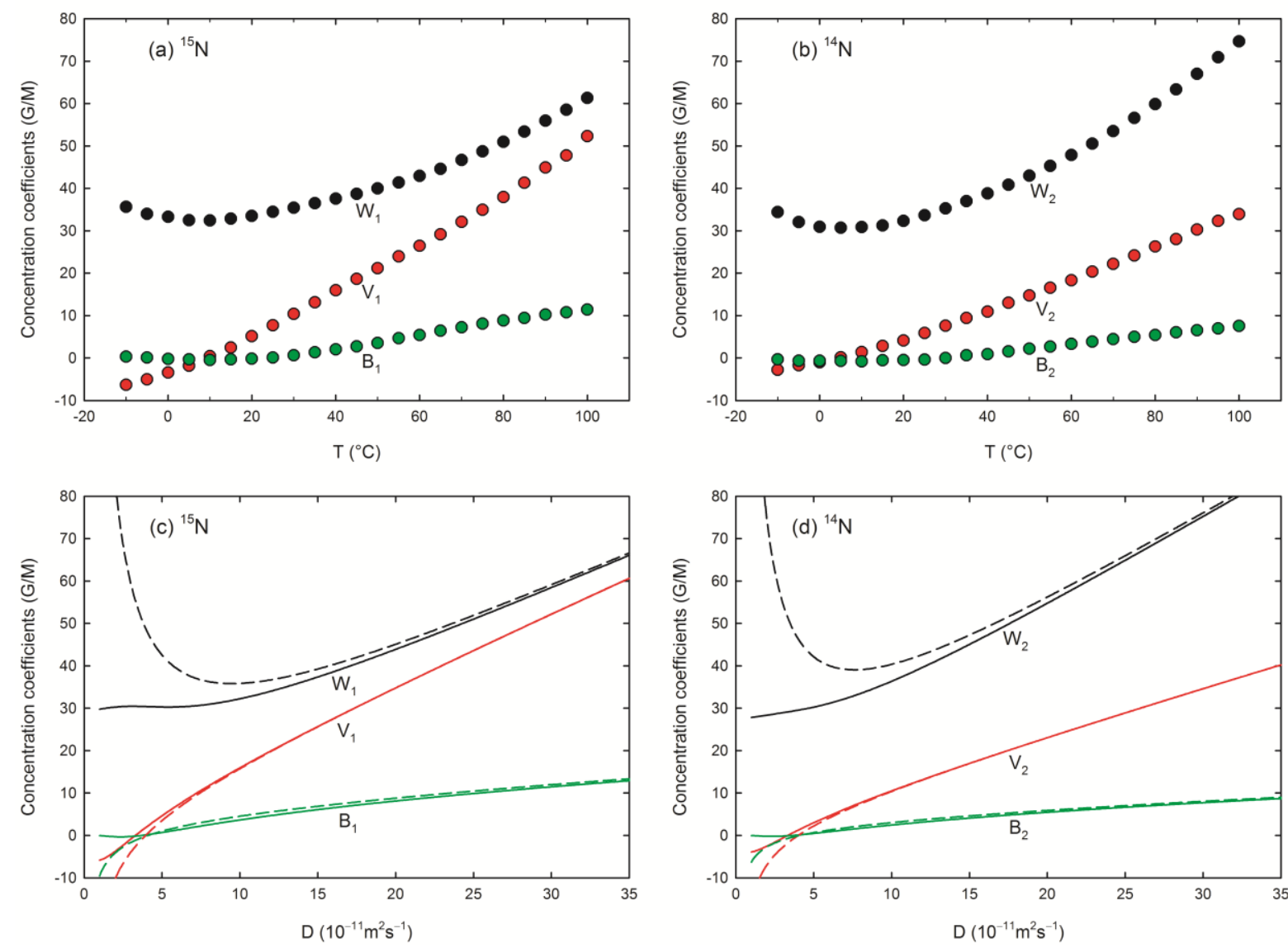

Figure 3. Concentration coefficients $W_{j}, V_{j}$, and $B_{j}$ as a function of temperature and diffusivity. The experimental temperature dependence of the concentration coefficients for (a) ${ }^{15} \mathrm{~N}$ - and (b) ${ }^{14} \mathrm{~N}$ pDTEMPONE in $\mathrm{C}_{2} \mathrm{C}_{1}$ ImTFSI. The theoretical diffusivity dependence of the coefficients for a spherical nitroxyl radical of radius $3.5 \AA$ having hyperfine splittings $A=22 \mathrm{G}$ for ${ }^{15} \mathrm{~N}$ and $A=16 \mathrm{G}$ for ${ }^{14} \mathrm{~N}$. The theoretical dependences are calculated numerically (full lines) and approximately using the first iteration solutions (dashed lines).

At low radical concentrations, the EPR parameters $\Gamma, \Lambda$, and $A$ exhibit linear concentration dependences due to HSE and DD interactions [7], which can be written as:

$$
\Gamma=\Gamma_{0}+W_{j} C ; \Lambda=\Lambda_{0}+V_{j} C ; A=A_{0}-B_{j} C .
$$

Here, $W_{j}, V_{j}$, and $B_{j}$ are the linear concentration coefficients of the average linewidth, coherence transfer rate, and hyperfine splitting, respectively, while the index $j=2 I$ has value 1 for ${ }^{15} \mathrm{~N}$-and 2 
for ${ }^{14} \mathrm{~N}$-pDTEMPONE [7]. The parameters $\Gamma, \Lambda$, and $A$ indeed show a linear dependence on $C$ in the measured concentration range (Fig. 2c-d), implying that the concentration coefficients can be evaluated as the slopes of the linear fits. The linear fits and concentration coefficients are obtained by the weighted linear regression method, where the weights are the inverse squares of the standard errors from the fitting procedure. By repeating the fitting procedure for EPR spectra and the linear regression method for the concentration dependences of the fitted parameters, the concentration coefficients $W_{j}, V_{j}$, and $B_{j}$ for ${ }^{15} \mathrm{~N}$ - and ${ }^{14} \mathrm{~N}$-pDTEMPONE are obtained at each measured temperature (Fig. 3). The concentration coefficients are analyzed by the model presented in the following section 3 .

\section{Theory}

\subsection{Model}

The radicals A and B subjected to an external magnetic field in solution are supposed to differ only in their Zeeman frequencies $\omega_{\mathrm{A}, \mathrm{B}}$. The radicals are modeled as hard spheres of the radius $\sigma / 2$, where $\sigma$ is the closest distance of the hard-core pair potential. The radius of pDTEMPONE is set to its van der Waals radius having the value of $3.5 \AA$ [20]. Assuming that the radicals continuously diffuse in the medium with the diffusivity $D$, the relative motion of a radical pair is characterized by the relative diffusion coefficient $D_{r}=2 D$, while the encounter time of a pair is given by $\tau_{\mathrm{D}}=\sigma^{2} / D_{r}$ [16]. In the HSE interaction having the form $H_{\mathrm{HSE}}=\hbar J(r) \vec{S}_{\mathrm{A}} \vec{S}_{\mathrm{B}}$, the exchange integral $J(r)$ strongly decreases with the relative distance $r$ between radicals. Hence, $J(r)$ is approximated by the function having a constant value $J_{0}$ in a narrow interaction layer and zero value outside of it. The interaction layer covers relative distances in the range $\sigma \leq r \leq \sigma+\Delta$, where a small interaction layer width, $\Delta$, satisfies $\Delta / \sigma<<1$. The values of $J_{0}$ and the contact time of radicals in the interaction layer $\tau_{\mathrm{C}}=\sigma \Delta / D_{r}$ are assumed to be large enough for pDTEMPONE to 
fulfill the strong HSE regime condition $J_{0} \tau_{\mathrm{C}}>>1$ [16]. Assuming that the encounter time $\tau_{\mathrm{D}}$ is so long that it satisfies $\tau_{\mathrm{D}} \omega_{\mathrm{A}, \mathrm{B}}>>1$, the DD interaction affects EPR spectra only by modulation of its secular part $H_{\mathrm{DD}}^{(0)}=\hbar \omega_{\mathrm{DD}}(\sigma / r)^{3} Y_{2}^{0}(\Omega)\left(S_{\mathrm{A}}^{+} S_{\mathrm{B}}^{-}+S_{\mathrm{A}}^{-} S_{\mathrm{B}}^{+}-4 S_{\mathrm{A}}^{z} S_{\mathrm{B}}^{z}\right)$. Here, $\Omega$ denotes the orientation angles of relative position vector $\vec{r}$ with respect to the magnetic field and $\omega_{\mathrm{DD}}=\sqrt{\pi / 5}\left(\hbar \gamma_{e}^{2} \mu_{0}\right) /\left(4 \pi \sigma^{3}\right)$ defines the characteristic DD frequency. The last assumption of the model is that the contact time $\tau_{\mathrm{C}}$ is short enough to satisfy $\omega_{\mathrm{DD}} \tau_{\mathrm{C}}<<1$ and $|\delta| \tau_{\mathrm{C}}<<1$, where $\delta=\omega_{\mathrm{A}}-\omega_{\mathrm{B}}$ is the difference in Zeeman frequencies of the radicals. The frequency differences between the radicals $\mathrm{A}$ and $\mathrm{B}$ can be $\delta=0, \pm a$ for the ${ }^{15} \mathrm{~N}-\mathrm{pDTEMPONE}$ solution, and $\delta=0, \pm a, \pm 2 a$ for the ${ }^{14} \mathrm{~N}$-pDTEMPONE solution. The values of frequency differences were set by taking typical values of the nitrogen hyperfine splitting $A=22 \mathrm{G}$ for ${ }^{15} \mathrm{~N}$ - and $A=16 \mathrm{G}$ for ${ }^{14} \mathrm{~N}$ pDTEMPONE.

\subsection{Evaluating the effects of spin interactions on the concentration coefficients}

We applied the formalism of the kinetic equations for the spin density matrices of radical pairs $[6,16,21]$ for the system of radicals described in the previous section. The concentration coefficients in G/M units were found to have the following form for the ${ }^{15} \mathrm{~N}$-pDTEMPONE solution [19]:

$$
\begin{aligned}
& W_{1}=\frac{10^{3} N_{\mathrm{A}}}{2 \gamma_{e}}\left\{k_{\mathrm{D}} \operatorname{Re}[p(a)]+\frac{\kappa_{\mathrm{DD}}^{2}}{k_{\mathrm{D}}} \operatorname{Re}\left[j_{\gamma}(0)+j_{\lambda}(0)+j_{\gamma}(a)\right]\right\} \\
& V_{1}=\frac{10^{3} N_{\mathrm{A}}}{2 \gamma_{e}}\left\{k_{\mathrm{D}} \operatorname{Re}[p(a)]-\frac{\kappa_{\mathrm{DD}}^{2}}{k_{\mathrm{D}}} \operatorname{Re}\left[j_{\lambda}(a)\right]\right\} \\
& B_{1}=-\frac{10^{3} N_{\mathrm{A}}}{\gamma_{e}}\left\{k_{\mathrm{D}} \operatorname{Im}[p(a)]+\frac{\kappa_{\mathrm{DD}}^{2}}{k_{\mathrm{D}}} \operatorname{Im}\left[j_{\gamma}(a)\right]\right\}
\end{aligned}
$$

and the following form for the ${ }^{14} \mathrm{~N}$-pDTEMPONE solution: 


$$
\begin{aligned}
& W_{2}=\frac{10^{3} N_{\mathrm{A}}}{3 \gamma_{e}}\left\{k_{\mathrm{D}} \operatorname{Re}\left[\frac{4 p(a)+2 p(2 a)}{3}\right]+\frac{\kappa_{\mathrm{DD}}^{2}}{k_{\mathrm{D}}} \operatorname{Re}\left[j_{\gamma}(0)+j_{\lambda}(0)+\frac{4 j_{\gamma}(a)+2 j_{\gamma}(2 a)}{3}\right]\right\} \\
& V_{2}=\frac{10^{3} N_{\mathrm{A}}}{3 \gamma_{e}}\left\{k_{\mathrm{D}} \operatorname{Re}\left[\frac{2 p(a)+p(2 a)}{3}\right]-\frac{\kappa_{\mathrm{DD}}^{2}}{k_{\mathrm{D}}} \operatorname{Re}\left[\frac{2 j_{\lambda}(a)+j_{\lambda}(2 a)}{3}\right]\right\} \\
& B_{2}=-\frac{10^{3} N_{\mathrm{A}}}{3 \gamma_{e}}\left\{k_{\mathrm{D}} \operatorname{Im}[p(a)+p(2 a)]+\frac{\kappa_{\mathrm{DD}}^{2}}{k_{\mathrm{D}}} \operatorname{Im}\left[j_{\gamma}(a)+j_{\gamma}(2 a)\right]\right\}
\end{aligned}
$$

In Eqs. (3a-b), $N_{\mathrm{A}}$ is the Avogadro constant, $k_{\mathrm{D}}=4 \pi \sigma D_{r}$ is the rate constant of diffusion encounters, and $\kappa_{\mathrm{DD}}=2 \sqrt{\pi} \sigma^{3} \omega_{\mathrm{DD}}$ is the rate constant of the DD interaction, while the complex parameters are given by [19]:

$$
\begin{aligned}
& p(\delta)=\frac{p_{1}(\delta)+p_{1}^{*}(-\delta)}{2} ; p_{1}(\delta)=\left.\frac{1}{4 \pi} \int \frac{\partial T_{1}(x, \Omega)}{\partial x}\right|_{x=1} d \Omega \\
& j_{\gamma}(\delta)=2 j_{1}(\delta)+j_{2}(\delta) ; j_{\lambda}(\delta)=j_{1}(\delta)+2 j_{2}(\delta) \\
& j_{1,2}(\delta)=\frac{q_{1,2}(\delta)+q_{1,2}^{*}(-\delta)}{2} ; q_{1,2}(\delta)=\frac{1}{i \beta_{\mathrm{D}}} \int d \Omega Y_{2}^{0}(\Omega) \int_{1}^{\infty} \frac{T_{1,2}(x, \Omega) d x}{x}
\end{aligned}
$$

Here, $x=r / \sigma(1<x<\infty)$ is the relative distance variable, $\beta_{\mathrm{D}}=\omega_{\mathrm{DD}} \tau_{\mathrm{D}}$ is a dimensionless DD parameter, and the functions $T_{1,2}(x, \Omega)$ represent non-zero elements of the correlation operator for the pair density matrix. These functions satisfy the following partial differential equations:

$$
\begin{aligned}
& \frac{1}{x} \frac{\partial^{2}\left(x T_{1}\right)}{\partial x^{2}}+\frac{\nabla_{\Omega}^{2} T_{1}}{x^{2}}=-i \beta_{\mathrm{D}} Y_{2}^{0}(\Omega) \frac{2 T_{1}+T_{2}}{x^{3}} \\
& \frac{1}{x} \frac{\partial^{2}\left(x T_{2}\right)}{\partial x^{2}}+\frac{\nabla_{\Omega}^{2} T_{2}}{x^{2}}+i \delta \tau_{\mathrm{D}} T_{2}=-i \beta_{\mathrm{D}} Y_{2}^{0}(\Omega) \frac{T_{1}+2 T_{2}}{x^{3}},
\end{aligned},
$$

where $\nabla_{\Omega}^{2}$ is the angular part of Laplacian. The boundary conditions for $x=1$ are $T_{1}=T_{2}$ and $\partial T_{1} / \partial x=-\partial T_{2} / \partial x$, while those for $x \rightarrow \infty$ are $T_{1} \rightarrow 1$ and $T_{2} \rightarrow 0$. Solving Eq. (3d) in the first iteration $(\mathrm{FI})$, the following relations for the parameters in Eq (3c) were derived [19]:

$$
p(\delta)=\frac{1+y}{2+y} ; j_{1}(\delta)=\frac{15+10 y+2 y^{2}}{9\left(6+4 y+y^{2}\right)} ; j_{2}(\delta)=\frac{1}{6+4 y+y^{2}},
$$


where $y^{2}=-i \delta \tau_{\mathrm{D}}$. Introducing the FI relations (Eq. 4) into Eqs. 3(a-c), the concentration coefficients $W_{j}, V_{j}$, and $B_{j}$ of ${ }^{15} \mathrm{~N}$ - and ${ }^{14} \mathrm{~N}$-pDTEMPONE were calculated as functions of radical diffusivity and presented by the dashed lines in Fig. 3c-d. In order to validate the FI relations, the diffusivity dependences of the concentration coefficients were also calculated by numerical solving of Eq. (3d), as described in Appendix. These exact results are presented by the full lines in Fig. 3c-d. As can be seen, the coefficients of spin dephasing or broadening coefficients $W_{j}$ have the highest values, but the exact and FI results differ most strongly. On the other hand, the exact and FI results for the hyperfine-splitting coefficients $B_{j}$ are close to each other, but the small values of these coefficients make them impractical for calculating the diffusivity in comparison with the other two. It can also be seen that the broadening coefficients $W_{j}$ saturate and become insensitive to the changes in diffusivity below the value of about $10 \times 10^{-11} \mathrm{~m}^{2} \mathrm{~s}^{-1}$, while the coherence-transfer coefficients $V_{j}$ remain sensitive to the smaller values of diffusivity. Additionally, the exact and FI results for the coefficients $V_{j}$ hardly differ above the value of about $5 \times 10^{-11} \mathrm{~m}^{2} \mathrm{~s}^{-1}$. The above analysis implies that the coherence-transfer coefficient is the best candidate for the calculation of the radical diffusivity.


Figure 4. Interrelations between the concentration coefficients. The dependences of $W_{j}$ and $B_{j}$ on $V_{j}$ for (a) ${ }^{15} \mathrm{~N}$ - and (b) ${ }^{14} \mathrm{~N}$-pDTEMPONE in $\mathrm{C}_{2} \mathrm{C}_{1}$ ImTFSI (circles), propylene carbonate (triangles), and ethylene glycol (squares). The lines denote the theoretical dependences for a spherical nitroxyl radical of radius $3.5 \AA$ with hyperfine splittings $A=22 \mathrm{G}$ for ${ }^{15} \mathrm{~N}$ and $A=16 \mathrm{G}$ for ${ }^{14} \mathrm{~N}$. 
In order to test our model, we compared the theoretical and experimental interrelations between different concentration coefficients. The experimental dependences of $W_{j}$ and $B_{j}$ on $V_{j}$ for ${ }^{15} \mathrm{~N}$ - and ${ }^{14} \mathrm{~N}$-pDTEMPONE in all studied liquids are compared with the theoretical dependences (Fig. 4). We can see that agreement between the theoretical and experimental dependences is quite good, which justifies the used model and allows us to calculate the diffusivities of both radicals from the theoretical dependences of $V_{j}$ in Fig. 3c-d.

\section{Results and discussion}

The values of radical diffusivity $D$ were obtained from the experimental values of coefficients $V_{j}$ and the numerically calculated theoretical diffusivity dependences of $V_{j}$ (Fig. 3). In the Stokes-Einstein plot (Fig. 5), the obtained values of $D$ were presented as a function of $T / \eta$, where $\eta$ is the viscosity of liquid. The viscosity values were obtained by fitting the experimental data for $\mathrm{C}_{2} \mathrm{C}_{1}$ ImTFSI [22] and EG [23] to a power-law temperature dependence. The viscosity values for PC were calculated from the published parameters of Vogel-Fulcher-Tamman (VFT) law [24]. Expectedly, the calculated diffusivities of ${ }^{15} \mathrm{~N}$ - and ${ }^{14} \mathrm{~N}$-pDTEMPONE are close to each other, justifying the applied experimental and theoretical methods.

The simplest model for prediction of tracer diffusivity is the Stokes-Einstein (SE) relation $D_{\mathrm{SE}}=k_{\mathrm{B}} T /\left(6 \pi \eta R_{\mathrm{U}}\right)$, where $R_{\mathrm{U}}$ is the radius of the solute molecule. Usually, the SE relation predicts the correct order of magnitude for the tracer diffusivity, and it tends to be more accurate as the tracer molecule becomes larger than the solvent molecule [3]. The SE diffusivity $D_{\mathrm{SE}}$ for pDTEMPONE is calculated by taking $R_{\mathrm{U}}=3.5 \AA$ (Fig. 5). It can be seen that the radical diffusivity values generally exceed those predicted by the SE relation. This effect, which can be quantified by the diffusion ratio $D / D_{\mathrm{SE}}$, is lowest for PC, medium for EG, and highest for $\mathrm{C}_{2} \mathrm{C}_{1} \mathrm{ImTFSI}$. Such an order of solvents 
can be illustrated by calculating the values of the diffusion ratio $D / D_{\mathrm{SE}}$ at $25^{\circ} \mathrm{C}$ ( $D$ is the average of ${ }^{15} \mathrm{~N}$ - and ${ }^{14} \mathrm{~N}$-pDTEMPONE), which are found to be 1.1 for PC, 2.3 for EG, and 3.4 for $\mathrm{C}_{2} \mathrm{C}_{1} \operatorname{ImTFSI}$. This diffusion ratio for neutral solutes was found to decrease with the relative size of van der Waals volumes of the solute $\left(V_{\mathrm{U}}\right)$ and solvent $\left(V_{\mathrm{V}}\right)$ molecules [3]. Using the method for the fast calculation of van der Waals volumes [25], we obtained the values $V_{\mathrm{U}}=177 \AA^{3}$ for pDTEMPONE, $V_{\mathrm{V}}=89 \AA^{3}$ for PC, and $V_{\mathrm{v}}=61 \AA^{3}$ for EG, while the value $V_{\mathrm{v}}=138 \AA^{3}$ for $\mathrm{C}_{2} \mathrm{C}_{1}$ ImTFSI was obtained as the average cation and anion volume in $\mathrm{C}_{2} \mathrm{C}_{1}$ ImTFSI [3]. It can be noticed that the ratio $V_{\mathrm{U}} / V_{\mathrm{v}}$ is smaller for PC than for EG, suggesting that $D / D_{\mathrm{SE}}$ should be larger for PC than for EG, while the opposite is observed.


Figure 5. Stokes-Einstein plots of radical diffusivity. The diffusivity of ${ }^{15} \mathrm{~N}$ - and ${ }^{14} \mathrm{~N}-\mathrm{pDTEMPONE}$ in $\mathrm{C}_{2} \mathrm{C}_{1}$ ImTFSI, propylene carbonate, and ethylene glycol. The dashed lines denote the StokesEinstein relations. The full lines are fits to Eq. (6). 
In order to take into account the type of solvent in the diffusion ratio, we applied the phenomenological relation that was used to fit the room-temperature diffusion data of various neutral tracers in various solvents [3]. The relation has the form:

$$
D / D_{\mathrm{SE}}=1+a\left(V_{\mathrm{U}} / V_{\mathrm{V}}\right)^{-p}
$$

where the fitted values of the positive parameters $a$ and $p$ differ for the ionic liquid, non-polar, and alcohol solvents. Using Eq. (5), we found the values $D / D_{\mathrm{SE}}=1.4$ for PC with the parameters for nonpolar solvents, $D / D_{\mathrm{SE}}=1.8$ for EG with the parameters for alcohol solvents, and $D / D_{\mathrm{SE}}=2.2$ for $\mathrm{C}_{2} \mathrm{C}_{1}$ ImTFSI with the parameters for ionic liquid solvents. These results predict a correct order of solvents in the observed values of $D / D_{\mathrm{SE}}$ at $25^{\circ} \mathrm{C}$, implying that the deviations from the SE law not only depend on the relative sizes of solute and solvent molecules but also on their relative types [3]. In the case of similar solute and solvent molecules, the smaller the difference between solute-solvent and solvent-solvent interactions, the smaller the deviation from the SE law. Here, the difference between neutral pDTEMPONE and the solvent molecules is lowest in PC, medium in EG having hydrogen-bonding molecules, and highest in $\mathrm{C}_{2} \mathrm{C}_{1}$ ImTFSI having charged molecules. The results of the molecular dynamics simulation of the diffusion of small neutral tracer in ionic liquid [26] suggest that the positive deviations of tracer diffusivity from the SE relation are additionally caused by the structural duality (heterogeneity) of ionic liquids, which consist of ionic ("stiff") and apolar ("soft") components. The "soft" component associated with the cationic apolar tails is more mobile and thus it is coupled to the tracer motion more than the "stiff" component. Hence, a tracer diffusing through the "soft" component makes its average diffusivity faster than expected from the SE relation.

Since the values of $D / D_{\mathrm{SE}}$ increase with lowering the temperature (Fig. 5), the temperature effect on the deviation from the SE law should also be analyzed. The average diffusivities of ${ }^{15} \mathrm{~N}$ and ${ }^{14} \mathrm{~N}$-pDTEMPONE were fitted to the empirical power-law dependence [27] of the form: 
$D\left(10^{-11} \mathrm{~m}^{2} \mathrm{~s}^{-1}\right)=D_{0}\left[\frac{T(\mathrm{~K})}{\eta(\mathrm{mPas})}\right]^{P}$,

resulting in the fitting curves shown in Fig. 5. The fitting procedure gives similar values of the exponent $P$ for all liquids ( 0.74 for $\mathrm{C}_{2} \mathrm{C}_{1}$ ImTFSI, 0.73 for PC, and 0.78 for EG), while the value of the prefactor $D_{0}$ is about $50 \%$ larger in $\mathrm{C}_{2} \mathrm{C}_{1} \operatorname{ImTFSI}(1.29)$ than in PC (0.86) and EG (0.85). The increase of $D_{0}$ from PC to $\mathrm{C}_{2} \mathrm{C}_{1} \operatorname{ImTFSI}$ agrees with the proposed relation $D_{0} \propto\left(V_{\mathrm{V}} / V_{\mathrm{U}}\right)^{\beta}$ where $\beta \approx 1$ [27] because the volume $V_{\mathrm{v}}$ of $\mathrm{C}_{2} \mathrm{C}_{1}$ ImTFSI is about $55 \%$ larger than that of PC. However, the volume of EG is about $68 \%$ of that for PC while $D_{0}$ is practically the same for both liquids, which indicates that the relative types of solute and solvent also play some role.

In order to further examine the temperature dependence of the tracer diffusivities of ${ }^{15} \mathrm{~N}$ - and ${ }^{14} \mathrm{~N}$-pDTEMPONE, their values are compared to the self-diffusivity values of each solvent obtained by NMR measurements (Fig. 6). The self-diffusivity values for the cation $\mathrm{C}_{2} \mathrm{C}_{1} \mathrm{Im}$ and the anion TFSI in $\mathrm{C}_{2} \mathrm{C}_{1}$ ImTFSI were calculated from the published parameters of the VFT law [28]. The selfdiffusivity data for PC were obtained by fitting the experimental data [1] to the VFT law, and those for EG were calculated from the published Arrhenius parameters [29].

Another model for the prediction of tracer diffusivity near room temperature for various solutes in organic solvents and water was proposed in Ref. [2]. The model predicts that the ratio between the tracer diffusivity $D$ and the self-diffusivity $D_{\mathrm{SD}}$ satisfies:

$$
\frac{D}{D_{\mathrm{SD}}}=\left(\frac{2}{1+R_{\mathrm{U}} / R_{\mathrm{V}}}\right)^{2}\left[\frac{1}{2}\left(1+\frac{M_{\mathrm{V}}}{M_{\mathrm{U}}}\right)\right]^{1 / 2}
$$

where $R_{\mathrm{U}}$ and $R_{\mathrm{V}}$ are van der Waals radii of the solute and solvent molecules, respectively, while $M_{\mathrm{U}}$ and $M_{\mathrm{V}}$ are the corresponding molecular masses. 

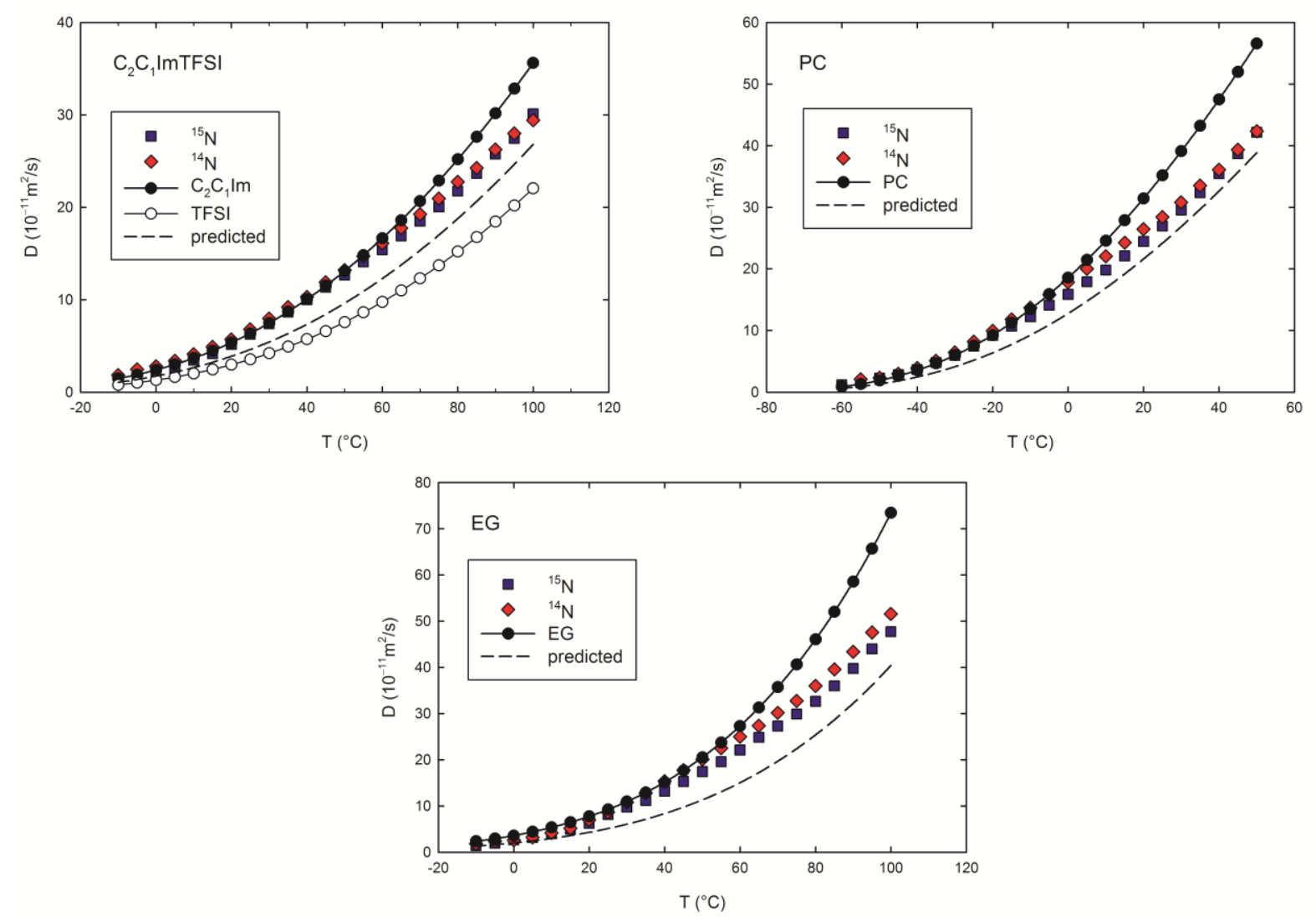

Figure 6. Tracer diffusivities of ${ }^{15} \mathrm{~N}$ - and ${ }^{14} \mathrm{~N}$-pDTEMPONE compared to the self-diffusivity in $\mathrm{C}_{2} \mathrm{C}_{1}$ ImTFSI, propylene carbonate, and ethylene glycol. The dashed lines represent the tracer diffusivities calculated by Eq. (7).

The parameters that describe the solute pDTEMPONE molecule in Eq. (7) are $R_{\mathrm{U}}=3.5 \AA$ and $M_{\mathrm{U}}=186.8 \mathrm{~g} / \mathrm{mol}$, which is the average value of the molar mass of ${ }^{15} \mathrm{~N}$ - and ${ }^{14} \mathrm{~N}-\mathrm{pDTEMPONE}$. Using the formula for van der Waals radii $R_{\mathrm{V}}=\left(3 V_{\mathrm{V}} / 4 \pi\right)^{1 / 3}$, we obtained $R_{\mathrm{V}}=3.2 \AA^{3}$ for $\mathrm{C}_{2} \mathrm{C}_{1} \operatorname{ImTFSI}, R_{\mathrm{V}}=2.8 \AA$ for PC, and $R_{\mathrm{V}}=2.4 \AA^{3}$ for EG. The average cation and anion mass in $\mathrm{C}_{2} \mathrm{C}_{1} \operatorname{ImTFSI}$ is $M_{\mathrm{v}}=195.7 \mathrm{~g} / \mathrm{mol}$, while the molar masses of PC and EG are $M_{\mathrm{V}}=102.1 \mathrm{~g} / \mathrm{mol}$ and $M_{\mathrm{V}}=62.1 \mathrm{~g} / \mathrm{mol}$, respectively. By inserting the above parameters in Eq. (7), we obtain the following predictions of $D / D_{\mathrm{SD}}=0.93$ for $\mathrm{C}_{2} \mathrm{C}_{1} \operatorname{ImTFSI}, D / D_{\mathrm{SD}}=0.69$ for PC, and $D / D_{\mathrm{SD}}=0.55$ for EG. The predicted tracer diffusivities are calculated from these ratios and the experimental self-diffusivities 
(Fig. 6). In the calculations, the self-diffusivity of $\mathrm{C}_{2} \mathrm{C}_{1}$ ImTFSI was taken as the average cation and anion diffusivities. The predictions work well at the highest measured temperatures in all liquids, but positive deviations from the predictions appear by decreasing the temperature (Fig. 6). The radical diffusivity in $\mathrm{C}_{2} \mathrm{C}_{1}$ ImTFSI tends to have the values of cation diffusivity, while the radical diffusivities in PC and EG tend to have the values of the corresponding self-diffusivities. The former result agrees with the coupling between the diffusive motions of a neutral tracer and cationic apolar tails, as indicated by molecular dynamics simulations in ionic liquid [26]. The latter results could indicate that such a coupling between the diffusive motion of tracer and original molecules exist in other liquids. These results need further examination, but they suggest that diffusive motion in a liquid becomes more cooperative and collective by decreasing the temperature. Such behavior of diffusive motion in glass-forming liquids has been evidenced by molecular dynamics simulations [30] and experiments [31,32].

\section{Conclusions}

With the aim to study radical diffusion in viscous liquids by EPR, we performed temperature dependent EPR measurements of the ${ }^{14} \mathrm{~N}$ - and ${ }^{15} \mathrm{~N}$-pDTEMPONE radicals in $\mathrm{C}_{2} \mathrm{C}_{1}$ ImTFSI ionic liquid, propylene carbonate, and ethylene glycol. In order to obtain the linear concentration coefficients of radicals' EPR parameters, the EPR spectra for various radical concentrations were fitted by the original spectral function derived from the modified Bloch equations. The calculated concentration coefficients of the average linewidth, coherence transfer rate, and hyperfine splitting were related to the radical diffusivity by using the kinetic equations for the spin evolution of a radical pair with the HSE and DD spin interactions. The radicals were modeled as continuously diffusing spherical objects in the hard-core pair potential, where the closest distance was set to twice the van der Waals radius of the radical, while the kinetic equations were solved numerically and approximately in the first iteration. The theoretical relations between the concentration coefficients 
and the radical diffusivity imply that the coherence-transfer coefficient is the most appropriate coefficient for calculation of radical diffusivity. Its values are comparable to the values of linewidth coefficient, but it stays sensitive to diffusivity down to smaller diffusivity values. Also, a practical advantage is that the difference between the numerically and approximately calculated relations is smaller for the coherence-transfer than the linewidth coefficient. Our model was tested by comparison of the theoretical and experimental interrelations between different concentration coefficients, which showed a good agreement.

By calculating the radical diffusivities from the coherence-transfer coefficients, the diffusivities of ${ }^{15} \mathrm{~N}$ - and ${ }^{14} \mathrm{~N}$-pDTEMPONE were found to be close to each other, which additionally justifies the applied experimental and theoretical methods. The calculated radical diffusivities generally exhibit positive deviations from the Stokes-Einstein prediction, which is the lowest for propylene carbonate, medium for ethylene glycol, and the highest for $\mathrm{C}_{2} \mathrm{C}_{1}$ ImTFSI. These deviations depend on the relative sizes of solute and solvent molecules, as well as on their relative types. The temperature dependencies of radical diffusivities in all three liquids were satisfactorily described by the fractional power-law modification of Stokes-Einstein law (6). We found similar values of the fractional exponent, but different values of the prefactor, which possibly reflects an influence of different relative sizes and types of solute and solvent molecules. The temperature dependencies of radical diffusivities in all liquids were further examined by comparing them to the temperature dependencies of the self-diffusivities from NMR measurements. We tested the model that predicts the tracer diffusivity from the self-diffusivity by only taking into account the relative sizes and masses of solute and solvent molecules. The model predicts well the radical diffusivity at the highest measured temperatures, but positive deviations from the predictions appear by lowering the temperature. The positive deviations seem to be due to the tendency of radical diffusivity to approach the values of cation diffusivity in $\mathrm{C}_{2} \mathrm{C}_{1}$ ImTFSI and the values of self-diffusivities in PC and EG. This effect for the solute molecule diffusing slower than the solvent molecule has not been noticed 
previously, as far as the authors are aware, and it could be one of the indicators of how diffusive motion in a given liquid can become more cooperative and collective in its nature.

\section{Acknowledgments}

This work has been supported by the Croatian Science Foundation [project numbers IP-2013-111108 and IP-2018-01-3168], as well as by the Foundation of the Croatian Academy of Sciences and Arts. D.M. wishes to thank Dejana Carić for her assistance in sample preparation. M.P. gratefully acknowledges support from NSF MRI Grant 1626632.

\section{References}

[1] O. Suárez-Iglesias, I. Medina, M. de los Ángeles Sanz, C. Pizarro, J. L. Bueno, Self-diffusion in molecular fluids and noble gases: available data, J. Chem. Eng. Data 60 (2015) 2757-2817, DOI: 10.1021/acs.jced.5b00323.

[2] K. Rah, S. Kwak, B. C. Eu, M. Lafleur, Relation of tracer diffusion coefficient and solvent selfdiffusion coefficient, J. Phys. Chem. A 106 (2002) 11841-11845, DOI: 10.1021/jp021659p.

[3] A. Kaintz, G. Baker, A. Benesi, M. Maroncelli, Solute diffusion in ionic liquids, NMR measurements and comparisons to conventional solvents, J. Phys. Chem. B 117 (2013) 11697-11708, DOI: 10.1021/jp405393d.

[4] A. Martinelli, M. Marechal, A. Ostlund, J. Cambedouzou, Insights into the interplay between molecular structure and diffusional motion in 1-alkyl-3-methylimidazolium ionic liquids: a combined PFG NMR and X-ray scattering study, Phys. Chem. Chem. Phys. 15 (2013) 5510-5517, DOI: 10.1039/C3CP00097D.

[5] X. Wang, Y. Chi, T. Mu, A review on the transport properties of ionic liquids, J. Mol. Liq. 193 (2014) 262-266, DOI: 10.1016/j.molliq.2014.03.011. 
[6] Yu. N. Molin, K. M. Salikhov, K. I. Zamaraev, Spin exchange principles and applications in chemistry and biology; Springer-Verlag: Berlin, 1980.

[7] K. M. Salikhov, Contributions of exchange and dipole-dipole interactions to the shape of EPR spectra of free radicals in diluted solutions, Appl. Magn. Reson. 38 (2010) 237-256, DOI: 10.1007/s00723-010-0128-x.

[8] B. Berner, D. Kivelson, The electron spin resonance line width method for measuring diffusion. A critique, J. Phys. Chem. 83 (1979) 1406-1412, DOI: 10.1021/j100474a012.

[9] A. M. Mastro, M. A. Babich, W. D. Taylor, A. D. Keith, Diffusion of a small molecule in the cytoplasm of mammalian cells, Proc. Natl. Acad. Sci. U. S. A. 81 (1984) 3414-3418, DOI: 10.1073/pnas.81.11.3414.

[10] J. Sachse, M. D. King, D. Marsh, ESR determination of lipid translational diffusion coefficients at low spin-label concentrations in biological membranes, using exchange broadening, exchange narrowing, and dipole-dipole interactions, J. Magn. Reson. (1969-1992) 71 (1987) 385-404, DOI: 10.1016/0022-2364(87)90241-1.

[11] A. Nayeem, S. B. Rananavare, V. S. S. Sastry, J. H. Freed, Heisenberg spin exchange and molecular diffusion in liquid crystals, J. Chem. Phys. 91 (1989) 6887-6905, DOI: 10.1063/1.457358.

[12] B. Y. Mladenova, N. A. Chumakova, V. I. Pergushov, A. I. Kokorin, G. Grampp, D. R. Kattnig, Rotational and translational diffusion of spin probes in room-temperature ionic liquids, J. Phys. Chem. B 116 (2012) 12295-12305, DOI: 10.1021/jp306583g.

[13] M. Wessig, M. Spitzbarth, M. Drescher, R. Winter, S. Polarz, Multiple scale investigation of molecular diffusion inside functionalized porous hosts using a combination of magnetic resonance methods, Phys. Chem. Chem. Phys. 17 (2015) 15976-15988, DOI: 10.1039/C5CP01369K.

[14] B. L. Bales, M. Peric, EPR line shifts and line shape changes due to spin exchange of nitroxide free radicals in liquids, J. Phys. Chem. B 101 (1997) 8707-8716, DOI: 10.1021/jp970995g. 
[15] B. L Bales, M. Meyer, S. Smith, M. Peric, EPR line shifts and line shape changes due to spin exchange of nitroxide-free radicals in liquids 4. Test of a method to measure re-encounter rates in liquids employing ${ }^{15} N$ and ${ }^{14} N$ nitroxide spin probes, J. Phys. Chem. A 112 (2008) 2177-2181, DOI: $10.1021 / j p 7107494$.

[16] K. M. Salikhov, The contribution from exchange interaction to line shifts in ESR spectra of paramagnetic particles in solutions, J. Magn. Reson. (1969-1992) 63 (1985) 271-279, DOI: 10.1016/0022-2364(85)90316-6.

[17] M. Peric, B. L. Bales, M. Peric, Electron paramagnetic resonance line shifts and line shape changes due to Heisenberg spin exchange and dipole-dipole interactions of nitroxide free radicals in liquids 8. Further experimental and theoretical efforts to separate the effects of the two interactions, J. Phys. Chem. A 116 (2012) 2855-2866, DOI: 10.1021/jp210057x.

[18] I. Peric, D. Merunka, B. L. Bales, M. Peric, Hydrodynamic and nonhydrodynamic contributions to the bimolecular collision rates of solute molecules in supercooled bulk water, J. Phys. Chem. B 118 (2014) 7128-7135, DOI: 10.1021/jp501330x.

[19] D. Merunka, M. Peric, Continuous diffusion model for concentration dependence of nitroxide EPR parameters in normal and supercooled water, J. Phys. Chem. B 121 (2017) 5259-5272, DOI: 10.1021/acs.jpcb.7b02550.

[20] I. Peric, D. Merunka, B. L. Bales, M. Peric, Rotation of four small nitroxide probes in supercooled bulk water, J. Phys. Chem. Lett. 4 (2013) 508-513, DOI: 10.1021/jz302107x.

[21] K. M. Salikhov, A. Ye. Mambetov, M. M. Bakirov, I. T. Khairuzhdinov, R. T. Galeev, R. B. Zaripov, B. L. Bales, Spin exchange between charged paramagnetic particles in dilute solutions, Appl. Magn. Reson. 45 (2014) 911-940, DOI: 10.1007/s00723-014-0571-1.

[22] A. Hofmann, M. Migeot, T. Hanemann, Investigation of binary mixtures containing 1-ethyl-3methylimidazolium bis(trifluoromethanesulfonyl)azanide and ethylene carbonate, J. Chem. Eng. 
Data 61 (2016) 114-123, DOI: 10.1021/acs.jced.5b00338; C. Schreiner, S. Zugmann, R. Hartl, H. J. Gores, Fractional Walden rule for ionic liquids: examples from recent measurements and a critique of the so-called ideal KCl line for the Walden plot, J. Chem. Eng. Data 55 (2010), 1784-1788, DOI: 10.1021/je900878j.

[23] R.-J. Lee, A. S. Teja, Viscosities of poly(ethylene glycols), J. Chem. Eng. Data 35 (1990) 385-387, DOI: 10.1021/je00062a003; P. J. Carvalho, C. H. G. Fonseca, M.-L. C. J. Moita, A. F. S. Santos, J. A. P. Coutinho, Thermophysical properties of glycols and glymes, J. Chem. Eng. Data 60 (2015) 3721-3737, DOI: 10.1021/acs.jced.5b00662; C. Yang, P. Ma, F. Jing, D. Tang, Excess molar volumes, viscosities, and heat capacities for the mixtures of ethylene glycol + water from $273.15 \mathrm{~K}$ to 353.15 K, J. Chem. Eng. Data 48 (2003) 836-840, DOI: 10.1021/je020140j; Dortmund Data Bank. http://www.ddbst.com/en/EED/PCP/VIS_C8.php (accessed 26 September 2018).

[24] F. Qi, K. U. Schug, S. Dupont, A. Döß, R. Böhmer, H. Sillescu, H. Kolshorn, H. Zimmermann, Structural relaxation of the fragile glass-former propylene carbonate studied by nuclear magnetic resonance, J. Chem. Phys. 112 (2000) 9455-9462, DOI: 10.1063/1.481588.

[25] Y. H. Zhao, M. H. Abraham, A. M. Zissimos, Fast calculation of van der Waals volume as a sum of atomic and bond contributions and its application to drug compounds, J. Org. Chem. 68 (2003) 7368-7373, DOI: 10.1021/jo034808o.

[26] J. C. Araque, S. K. Yadav, M. Shadeck, M. Maroncelli, C. J. Margulis, How is diffusion of neutral and charged tracers related to the structure and dynamics of a room-temperature ionic liquid? Large deviations from Stokes-Einstein behavior explained, J. Phys. Chem. B 119 (2015) 7015-7029, DOI: 10.1021/acs.jpcb.5b01093.

[27] Y. Kimura, Y. Kida, Y. Matsushita, Y. Yasaka, M. Ueno, K. Takahashi, Universality of viscosity dependence of translational diffusion coefficients of carbon monoxide, diphenylacetylene, 
and diphenylcyclopropenone in ionic liquids under various conditions, J. Phys. Chem. B 119 (2015) 8096-8103, DOI: 10.1021/acs.jpcb.5b02898.

[28] A. Noda, K. Hayamizu, M. Watanabe, Pulsed-gradient spin-echo ${ }^{1} H$ and ${ }^{19} F$ NMR ionic diffusion coefficient, viscosity, and ionic conductivity of non-chloroaluminate room-temperature ionic liquids, J. Phys. Chem. B 105 (2001) 4603-4610, DOI: 10.1021/jp004132q.

[29] K. H. Nothnagel, A. Weiss, Self-diffusion in liquids. III. Self-diffusion of ethylene glycol, $\mathrm{DOCH}_{2} \mathrm{CH}_{2} \mathrm{OD}$, and methanol, $\mathrm{CH}_{3} \mathrm{OD}$, in the binary liquid systems $\mathrm{DOCH}_{2} \mathrm{CH}_{2} \mathrm{OD}-\mathrm{CD}_{3} \mathrm{OD}$ and $\mathrm{DOCD}_{2} \mathrm{CD}_{2} \mathrm{OD}-\mathrm{CH}_{3} \mathrm{OD}$, Berich. Bunsen Gesell. 73 (1969) 1033-1040, DOI: 10.1002/bbpc. 19690731020.

[30] C. Donati, J. F. Douglas, W. Kob, S. J. Plimpton, P. H. Poole, S. C. Glotzer, Stringlike cooperative motion in a supercooled liquid, Phys. Rev. Lett. 80 (1998) 2338-2341, DOI: 10.1103/PhysRevLett.80.2338.

[31] H. Ehmler, A. Heesemann, K. Rätzke, F. Faupel, U. Geyer, Mass dependence of diffusion in a supercooled metallic melt, Phys. Rev. Lett. 80 (1998) 4919-4922, DOI: 10.1103/PhysRevLett.80.4919.

[32] A. Jaiswal, T. Egami, K. F. Kelton, K. S. Schweizer, and Y. Zhang, Correlation between fragility and the Arrhenius crossover phenomenon in metallic, molecular, and network liquids, Phys. Rev. Lett. 117 (2016) 205701-1-6, DOI: 10.1103/PhysRevLett.117.205701. 


\section{Appendix A. Setting up the equations}

Defining the frequency difference parameter $\alpha_{\mathrm{D}}=\delta \tau_{\mathrm{D}}$ and using the relation for spherical functions $Y_{L}^{0}(\Omega)=p_{L}(w) /(2 \pi)^{1 / 2}$, where $\quad w=\cos \Theta \quad$ and $\quad p_{L}(w)=(L+1 / 2)^{1 / 2} P_{L}(w) \quad$ is the normalized Legendre polynomial of $L$-th degree, Eqs. (3d) can be transformed into the form:

$$
\begin{aligned}
& \frac{\partial^{2}\left(x T_{1}\right)}{x \partial x^{2}}+\frac{\nabla_{w}^{2} T_{1}}{x^{2}}=-\frac{i \beta_{\mathrm{D}}}{\sqrt{2 \pi}} p_{2}(w) \frac{2 T_{1}+T_{2}}{x^{3}} \\
& \frac{\partial^{2}\left(x T_{2}\right)}{x \partial x^{2}}+\frac{\nabla_{w}^{2} T_{2}}{x^{2}}+i \alpha_{\mathrm{D}} T_{2}=-\frac{i \beta_{\mathrm{D}}}{\sqrt{2 \pi}} p_{2}(w) \frac{T_{1}+2 T_{2}}{x^{3}} . \\
& \nabla_{w}^{2} T_{i}=\frac{\partial}{\partial w}\left[\left(1-w^{2}\right) \frac{\partial T_{i}}{\partial w}\right]
\end{aligned}
$$

The boundary conditions (BCs) for $T_{1,2}(x, w)$ are:

$$
\begin{aligned}
& T_{1}(x \rightarrow \infty, w) \rightarrow 1 ; T_{2}(x \rightarrow \infty, w) \rightarrow 0 \\
& \left(\partial T_{1} / \partial x\right)_{1, w}=-\left(\partial T_{2} / \partial x\right)_{1, w} ; T_{1}(1, w)=T_{2}(1, w)
\end{aligned},
$$

while the parameters in Eq. (3c) can be written as:

$$
p_{1}(\delta)=\left.\frac{1}{2} \int_{-1}^{1} d w \frac{\partial T_{1}(x, w)}{\partial x}\right|_{1, w} ; q_{1,2}(\delta)=\frac{\sqrt{2 \pi}}{i \beta_{\mathrm{D}}} \int_{1}^{\infty} d x \int_{-1}^{1} d w p_{2}(w) \frac{T_{1,2}(x, w)}{x}
$$

We define the new variable $q=1 / x(0<q<1)$ and the new functions $F_{+}(q, w)=T_{+}(q, w)-1$ and $F_{-}(q, w)=T_{-}(q, w)-1+q$, where $T_{ \pm}(q, w)=T_{1}(q, w) \pm T_{2}(q, w)$. For these variables and functions, we get the following differential equations:

$$
\begin{aligned}
& q^{4} \frac{\partial^{2} F_{+}}{\partial q^{2}}+q^{2} \nabla_{w}^{2} F_{+}+i \alpha_{\mathrm{D}} \frac{F_{+}-F_{-}}{2}+\frac{3 i \beta_{\mathrm{D}}}{\sqrt{2 \pi}} p_{2}(w) q^{3} F_{+}=-\frac{3 i \beta_{\mathrm{D}}}{\sqrt{2 \pi}} p_{2}(w) q^{3}-i \alpha_{\mathrm{D}} \frac{q}{2} \\
& q^{4} \frac{\partial^{2} F_{-}}{\partial q^{2}}+q^{2} \nabla_{w}^{2} F_{-}-i \alpha_{\mathrm{D}} \frac{F_{+}-F_{-}}{2}+\frac{i \beta_{\mathrm{D}}}{\sqrt{2 \pi}} p_{2}(w) q^{3} F_{-}=-\frac{i \beta_{\mathrm{D}}}{\sqrt{2 \pi}} p_{2}(w) q^{3}(1-q)+i \alpha_{\mathrm{D}} \frac{q}{2}
\end{aligned},
$$

and the following BCs: 


$$
F_{+}(0, w)=\left(\partial F_{+} / \partial q\right)_{1, w}=0 ; F_{-}(0, w)=F_{-}(1, w)=0
$$

The parameters (A1c) take the form:

$$
\begin{aligned}
& p_{1}(\delta)=\frac{1}{4} \int_{-1}^{1} d w\left[1-\left.\frac{\partial F_{+}(w, q)}{\partial q}\right|_{1, w}-\left.\frac{\partial F_{-}(q, w)}{\partial q}\right|_{1, w}\right] \\
& q_{1,2}(\delta)=\sqrt{\frac{\pi}{2}} \frac{1}{i \beta_{\mathrm{D}}} \int_{0}^{1} d q \int_{-1}^{1} d w p_{2}(w) \frac{T_{+}(q, w) \pm T_{-}(q, w)}{q}
\end{aligned}
$$

We perform the expansion $F_{ \pm}(q, w)=\sum_{m=1}^{\infty} p_{2 m-2}(w) u_{m}^{ \pm}(q)$ in (A2a). Using the equality $\nabla_{w}^{2} p_{L}(w)=-L(L+1) p_{L}(w)$ and integrating both sides with $\int d w p_{2 l-2}(w)$, we get the following system of differential equations for radial functions $u_{l}^{ \pm}(q)$ :

$$
\begin{aligned}
& q^{4} u_{l}^{\prime \prime+}-L(L+1) q^{2} u_{l}^{+}+i \alpha_{\mathrm{D}} \frac{u_{l}^{+}-u_{l}^{-}}{2}+\frac{3 i \beta_{\mathrm{D}} q^{3}}{\sqrt{2 \pi}} \sum_{m=l-1}^{l+1} \varphi_{l m} u_{m}^{+}=-\frac{3 i \beta_{\mathrm{D}} q^{3}}{\sqrt{2 \pi}} \delta_{l, 2}-\frac{i \alpha_{\mathrm{D}} q}{\sqrt{2}} \delta_{l, 1} \\
& q^{4} u_{l}^{\prime \prime-}-L(L+1) q^{2} u_{l}^{-}-i \alpha_{\mathrm{D}} \frac{u_{l}^{+}-u_{l}^{-}}{2}+\frac{i \beta_{\mathrm{D}} q^{3}}{\sqrt{2 \pi}} \sum_{m=l-1}^{l+1} \varphi_{l m} u_{m}^{-}=-\frac{i \beta_{\mathrm{D}} q^{3}(1-q)}{\sqrt{2 \pi}} \delta_{l, 2}+\frac{i \alpha_{\mathrm{D}} q}{\sqrt{2}} \delta_{l, 1}
\end{aligned},
$$

where $u_{l}^{\prime \prime \pm}(q)=d^{2} u_{l}^{ \pm}(q) / d q^{2}, L=2 l-2$ and $\varphi_{l m}=\int d w p_{2 l-2}(w) p_{2 m-2}(w) p_{2}(w)$. The BCs are:

$$
u_{l}^{+}(0)=u_{l}^{\prime+}(1)=0 ; u_{l}^{-}(0)=u_{l}^{-}(1)=0
$$

The quantities (A2c) become:

$$
p_{1}(\delta)=\frac{1}{2}\left[1-\frac{u_{1}^{\prime+}(1)+u_{1}^{\prime-}(1)}{\sqrt{2}}\right] ; q_{1,2}(\delta)=\sqrt{\frac{\pi}{2}} \frac{1}{i \beta_{\mathrm{D}}} \int_{0}^{1} d q \frac{u_{2}^{+}(q) \pm u_{2}^{-}(q)}{q}
$$

The solutions of the differential equation:

$$
q^{4} v_{l}^{\prime \prime \pm}(q)-L(L+1) q^{2} v_{l}^{ \pm}(q)=-\lambda_{l}^{ \pm} q^{3} v_{l}^{ \pm}(q) ; L=2 l-2 \geq 0
$$

that satisfy $\mathrm{BCs}(\mathrm{A} 3 \mathrm{~b})$ at $q=0$, i.e., $v_{l}^{ \pm}(0)=0$ are given by $v_{l}^{ \pm}(q)=z J_{4 l-3}(z)$, where $z=2\left(\lambda_{l}^{ \pm} q\right)^{1 / 2}$ and $J_{4 l-3}(z)$ is Bessel functions of the first kind. In order to satisfy BCs (A3b) at $q=1$, we define 
sets of numbers $\lambda_{l j}^{+}=\left(s_{l j} / 2\right)^{2}$ and $\lambda_{l j}^{-}=\left(p_{l j} / 2\right)^{2}$ where $j=1,2,3 \ldots$, while $s_{l j}, p_{l j}>0$ satisfy the following relations $J_{4 l-3}\left(s_{l j}\right)+s_{l j} J_{4 l-3}^{\prime}\left(s_{l j}\right)=0$ and $J_{4 l-3}\left(p_{l j}\right)=0$. We construct the functions:

$$
\begin{aligned}
& v_{l j}^{+}(q)=n_{l j}^{+} s_{l j} \sqrt{q} J_{4 l-3}\left(s_{l j} \sqrt{q}\right) ; v_{l j}^{-}(q)=n_{l j}^{-} p_{l j} \sqrt{q} J_{4 l-3}\left(p_{l j} \sqrt{q}\right) \\
& n_{l j}^{+}=\frac{1}{\left|J_{4 l-3}\left(s_{l j}\right)\right| \sqrt{1+s_{l j}^{2}-(4 l-3)^{2}}} ; n_{l j}^{-}=\frac{1}{p_{l j}\left|J_{4 l-2}\left(p_{l j}\right)\right|}
\end{aligned}
$$

These functions satisfy the following conditions at $q=1$ :

$v_{l j}^{\prime+}(1)=n_{l j}^{+}\left(s_{l j} / 2\right)\left[J_{4 l-3}\left(s_{l j}\right)+s_{l j} J_{4 l-3}^{\prime}\left(s_{l j}\right)\right]=0 ; v_{l j}^{-}(1)=n_{l j}^{+} p_{l j} J_{4 l-3}\left(p_{l j}\right)=0$

and the orthogonality relationships:

$$
\int_{0}^{1} d q q^{-1} v_{l j}^{+}(q) v_{l k}^{+}(q)=\int_{0}^{1} d q q^{-1} v_{l j}^{-}(q) v_{l k}^{-}(q)=\delta_{l k}
$$

Thus, the functions $v_{l, j}^{ \pm}(q)$ form an orthonormal set with weight $q^{-1}$ and satisfy the same BCs (A3b) as $u_{l}^{ \pm}(q)$. Using the expansions $u_{l}^{+}(q)=\sum_{j=1}^{\infty} M_{l j} v_{l j}^{+}(q)$ and $u_{l}^{-}(q)=\sum_{j=1}^{\infty} N_{l j} v_{l j}^{-}(q)$, Eqs. (A3a) were transformed into the following sets of equations after dividing by $i$ :

$$
\begin{aligned}
& \sum_{j}\left[\frac{i s_{l j}^{2}}{4} q^{3} v_{l j}^{+} M_{l j}+\frac{\alpha_{\mathrm{D}}}{2}\left(v_{l j}^{+} M_{l j}-v_{l j}^{-} N_{l j}\right)+\frac{3 \beta_{\mathrm{D}}}{\sqrt{2 \pi}} \sum_{m=l-1}^{l+1} \varphi_{l m} q^{3} v_{m j}^{+} M_{m j}\right]=-\frac{3 \beta_{\mathrm{D}} q^{3}}{\sqrt{2 \pi}} \delta_{l, 2}-\frac{\alpha_{\mathrm{D}} q}{\sqrt{2}} \delta_{l, 1} \\
& \sum_{j}\left[\frac{i p_{l j}^{2}}{4} q^{3} v_{l j}^{-} N_{l j}-\frac{\alpha_{\mathrm{D}}}{2}\left(v_{l j}^{+} M_{l j}-v_{l j}^{-} N_{l j}\right)+\frac{\beta_{\mathrm{D}}}{\sqrt{2 \pi}} \sum_{m=l-1}^{l+1} \varphi_{l m} q^{3} v_{m j}^{-} N_{m j}\right]=-\frac{\beta_{\mathrm{D}} q^{3}(1-q)}{\sqrt{2 \pi}} \delta_{l, 2}+\frac{\alpha_{\mathrm{D}} q}{\sqrt{2}} \delta_{l, 1} \cdot(\mathrm{A} 8
\end{aligned}
$$

Integrating (A8a) and (A8b) with $\int_{0}^{1} d q q^{-1} v_{l k}^{+}(q)$ and $\int_{0}^{1} d q q^{-1} v_{l k}^{-}(q)$, respectively, we get the following linear equations for the coefficients $M_{l k}$ and $N_{l k}$ :

$$
\begin{aligned}
& \sum_{j} \frac{i s_{l j}^{2}}{4} D_{k j}^{+}(l, 0) M_{l, j}+\sum_{j} \frac{3 \beta_{\mathrm{D}}}{\sqrt{2 \pi}}\left[\varphi_{l, l} D_{k j}^{+}(l, 0) M_{l, j}+\varphi_{l, l+1} D_{k j}^{+}(l, 1) M_{l+1, j}+\varphi_{l-1, l} D_{j k}^{+}(l-1,1) M_{l-1, j}\right] \\
& +\frac{\alpha_{\mathrm{D}}}{2} M_{l, k}-\frac{\alpha_{\mathrm{D}}}{2} \sum_{j} A_{k j}(l) N_{l, j}=-\delta_{l, 2} \frac{3 \beta_{\mathrm{D}}}{\sqrt{2 \pi}} d_{k, 2}^{+}-\delta_{l, 1} \frac{\alpha_{\mathrm{D}}}{\sqrt{2}} a_{k}^{+}
\end{aligned}
$$




$$
\begin{aligned}
& \sum_{j} \frac{i p_{l j}^{2}}{4} D_{k j}^{-}(l, 0) N_{l, j}+\sum_{j} \frac{\beta_{\mathrm{D}}}{\sqrt{2 \pi}}\left[\varphi_{l, l} D_{k j}^{-}(l, 0) N_{l, j}+\varphi_{l, l+1} D_{k j}^{-}(l, 1) N_{l+1, j}+\varphi_{l-1, l} D_{j k}^{-}(l-1,1) N_{l-1, j}\right] \\
& +\frac{\alpha_{\mathrm{D}}}{2} N_{l, k}-\frac{\alpha_{\mathrm{D}}}{2} \sum_{j} A_{j k}(l) M_{l, j}=-\delta_{l, 2} \frac{\beta_{\mathrm{D}}}{\sqrt{2 \pi}} d_{k, 2}^{-}+\delta_{l, 2} \frac{\beta_{\mathrm{D}}}{\sqrt{2 \pi}} d_{k, 3}^{-}+\delta_{l, 1} \frac{\alpha_{\mathrm{D}}}{\sqrt{2}} a_{k}^{-}
\end{aligned} .
$$

The parameters in these equations are given by the following integrals:

$$
\begin{aligned}
& D_{k j}^{ \pm}(l, m)=\int_{0}^{1} d q q^{2} v_{l, k}^{ \pm}(q) v_{l+m, j}^{ \pm}(q) ; d_{k, n}^{ \pm}=\int_{0}^{1} d q v_{2, k}^{ \pm}(q) q^{n} \\
& A_{k j}(l)=\int_{0}^{1} d q q^{-1} v_{l, k}^{+}(q) v_{l, j}^{-}(q) ; a_{k}^{ \pm}=\int_{0}^{1} d q v_{1, k}^{ \pm}(q)
\end{aligned}
$$

\section{Appendix B. Solving the equations}

In order to numerically solve the set of linear equations (A9), we construct matrices of dimension $B \times B$, where $B=2 b n$. Here, $b$ and $n$ are the numbers of the used angular and radial basis functions, respectively, i.e, $l=1,2 \ldots, b$ and $k, j=1,2 \ldots, n$. Defining indices $m_{l, j}=b(l-1)+j$ and $n_{l, j}=b(n+l-1)+j$, the system of linear equations (A9) can be written in the matrix form:

$\mathbf{K s}=\mathbf{r}$,

where the components of column vector $\mathbf{s}$ are related to the unknown coefficients as:

$$
\mathbf{s}\left(m_{l, j}\right) \equiv M_{l, j} ; \mathbf{s}\left(n_{l, j}\right) \equiv N_{l, j} .
$$

The column vector $\mathbf{r}$ in (B1a) can be written as $\mathbf{r}=\alpha_{\mathrm{D}} \mathbf{r}_{\alpha}+\beta_{\mathrm{D}} \mathbf{r}_{\beta}$, where the non-zero components of $\mathbf{r}_{\alpha}$ and $\mathbf{r}_{\beta}$ are given by:

$$
\begin{aligned}
& \mathbf{r}_{\alpha}\left(m_{1, k}\right)=-(1 / \sqrt{2}) a_{k}^{+} ; \mathbf{r}_{\alpha}\left(n_{1, k}\right)=(1 / \sqrt{2}) a_{k}^{-} \\
& \mathbf{r}_{\beta}\left(m_{2, k}\right)=-(3 / \sqrt{2 \pi}) d_{k, 2}^{+} ; \mathbf{r}_{\beta}\left(n_{2, k}\right)=-(1 / \sqrt{2 \pi}) d_{k, 2}^{-}+(1 / \sqrt{2 \pi}) d_{k, 3}^{-}
\end{aligned}
$$


The matrix of the system $\mathbf{K}$ in (B1a) can be written as $\mathbf{K}=i \mathbf{K}_{I}+\alpha_{\mathrm{D}} \mathbf{K}_{\alpha}+\beta_{\mathrm{D}} \mathbf{K}_{\beta}$, where the matrices $\mathbf{K}_{I}, \mathbf{K}_{\alpha}$, and $\mathbf{K}_{\beta}$ have the following non-zero components:

$\mathbf{K}_{I}\left(m_{l, k}, m_{l, j}\right)=\left(s_{l j}^{2} / 4\right) D_{k j}^{+}(l, 0) ; \mathbf{K}_{I}\left(n_{l, k}, n_{l, j}\right)=\left(p_{l j}^{2} / 4\right) D_{k j}^{-}(l, 0)$,

$\mathbf{K}_{\alpha}\left(m_{l, k}, m_{l, k}\right)=\mathbf{K}_{\alpha}\left(n_{l, k}, n_{l, k}\right)=1 / 2$

$\mathbf{K}_{\alpha}\left(m_{l, k}, n_{l, j}\right)=\mathbf{K}_{\alpha}\left(n_{l, j}, m_{l, k}\right)=-(1 / 2) A_{k j}(l)^{\prime}$

$\mathbf{K}_{\beta}\left(m_{l, k}, m_{l, j}\right)=(3 / \sqrt{2 \pi}) \varphi_{l, l} D_{k j}^{+}(l, 0) ; \mathbf{K}_{\beta}\left(n_{l, k}, n_{l, j}\right)=(1 / \sqrt{2 \pi}) \varphi_{l, l} D_{k j}^{-}(l, 0)$

$\mathbf{K}_{\beta}\left(m_{l, k}, m_{l+1, j}\right)=\mathbf{K}_{\beta}\left(m_{l+1, j}, m_{l, k}\right)=(3 / \sqrt{2 \pi}) \varphi_{l, l+1} D_{k j}^{+}(l, 1)$

$\mathbf{K}_{\beta}\left(n_{l, k}, n_{l+1, j}\right)=\mathbf{K}_{\beta}\left(n_{l+1, j}, n_{l, k}\right)=(1 / \sqrt{2 \pi}) \varphi_{l, l+1} D_{k j}^{-}(l, 1)$

The HSE parameter in Eq. (A3c) takes the form:

$$
p_{1}(\delta)=\frac{1}{2}-\frac{1}{2} \sum_{k=1}^{\infty} v_{1, k}^{\prime-}(1) \frac{N_{1, k}\left(\alpha_{\mathrm{D}}, \beta_{\mathrm{D}}\right)}{\sqrt{2}}
$$

Using $v_{1 k}^{\prime-}(1)=-\left(p_{1 k} / 2\right) \operatorname{sgn} J_{2}\left(p_{1 k}\right)$, we get:

$$
p_{1}(\delta)=\frac{1}{2}+\frac{1}{4 \sqrt{2}} \sum_{k=1}^{\infty} p_{1, k} \operatorname{sgn} J_{2}\left(p_{1, k}\right) N_{1, k}\left(\alpha_{\mathrm{D}}, \beta_{\mathrm{D}}\right)
$$

The DD parameters in Eq. (A3c) take the form:

$q_{1,2}(\delta)=\sqrt{\frac{\pi}{2}} \frac{1}{i \beta_{\mathrm{D}}} \sum_{k=1}^{\infty}\left[d_{k,-1}^{+} M_{2, k}\left(\alpha_{\mathrm{D}}, \beta_{\mathrm{D}}\right) \pm d_{k,-1}^{-} N_{2, k}\left(\alpha_{\mathrm{D}}, \beta_{\mathrm{D}}\right)\right]$

where the parameters $d_{k,-1}^{ \pm}=\int_{0}^{1} d q v_{2, k}^{ \pm}(q) q^{-1}$ from Eq. (A9c) are given by:

$$
\begin{aligned}
& d_{k,-1}^{+} / 2=n_{2 k}^{+} \int_{0}^{s_{2 k}} d z J_{5}(z)=n_{2 k}^{+}\left[1+\left(1-8 / s_{2 k}^{2}\right) J_{2}\left(s_{2 k}\right)-10 J_{3}\left(s_{2 k}\right) / s_{2 k}\right] \\
& d_{k,-1}^{-} / 2=n_{2 k}^{-} \int_{0}^{p_{2 k}} d z J_{5}(z)=n_{2 k}^{-}\left[1+\left(1-8 / p_{2 k}^{2}\right) J_{2}\left(p_{2 k}\right)-10 J_{3}\left(p_{2 k}\right) / p_{2 k}\right]
\end{aligned}
$$


The complex parameters in Eqs. (3c) can be calculated from the HSE and DD parameters in Eqs. (B3) and (B4).

After defining the basis dimensions $b$ and $n$, we calculated the sets of numbers $s_{l j}$ and $p_{l j}$ using the Mathematica commands for finding the roots of Bessel functions and their derivative. Then, we constructed radial basis functions according to Eq. (A5) and calculated the parameters from Eq. (A9c) that gives non-zero-elements of the matrices and column vectors defined by Eqs. (B1). The calculated matrices $\mathbf{K}_{I}, \mathbf{K}_{\beta}$, and $\mathbf{K}_{\alpha}$ of dimension $B \times B$ and column vectors $\mathbf{r}_{\alpha}$ and $\mathbf{r}_{\beta}$ of dimension $B$ were saved for further calculations.
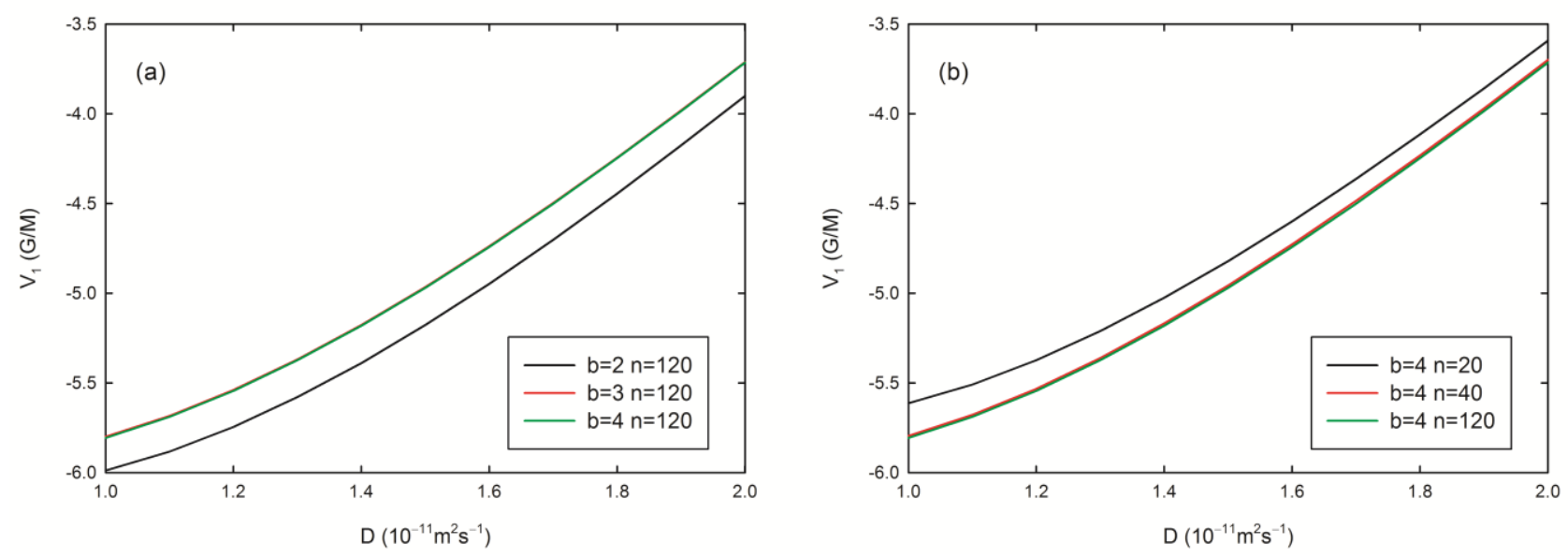

Figure B1. Numerically calculated concentration coefficient $V_{1}$ for ${ }^{15} \mathrm{~N}-\mathrm{pDTEMPONE}$ as a function of the basis dimensions $b$ and $n .{ }^{15} \mathrm{~N}$-pDTEMPONE is modeled as a spherical radical of radius $3.5 \AA$ having the hyperfine splitting $A=22 \mathrm{G}$.

Using the previously saved matrices and column vectors for particular numbers $b$ and $n$, the concentration coefficients $W_{j}, V_{j}$, and $B_{j}$ of ${ }^{15} \mathrm{~N}$ - or ${ }^{14} \mathrm{~N}$-labeled radicals were calculated in a separate procedure as a function of radical diffusivity. In the calculations, the minimum, maximum, and step values of diffusivity were defined, and the linear system (B1a) was solved for each value of diffusivity. The concentration coefficients were calculated from the linear system solution by using Eqs. (B3), (B4), and (3). Each calculation was performed at fixed values of the radius of radical $\sigma / 2$ 
and the nitrogen hyperfine splitting $A$. The convergence of the results with the basis dimensions $b$ and $n$ was checked for the ${ }^{15} \mathrm{~N}$-labeled radical (Fig. B1). It was found that $b=4$ and $n=120$ are large enough for our purposes. 